\title{
Self-reported hypoglycaemia in insulin-treated patients with diabetes mellitus: results from the Singapore cohort of the International Operations Hypoglycaemia Assessment Tool study
}

Ngiap Chuan Tan ${ }^{1}$, mBBS, MMed, Su-Yen Goh ${ }^{2}$, MBBS, MRCP, Eric Yin-Hao Khoo ${ }^{3}$, MBChB, FRCP, Rinkoo Dalan ${ }^{4}$, MBBS, FAMS, Agnes Koong ${ }^{1}$, MBBS, MmD, Chin Meng $\underline{K h o o}^{3}$, MBBS, MRCP, Teck Shi $\underline{T a n}^{1}$, MBBS, FRCoG, Anand B Jain ${ }^{5}$, MBBS, MD, Arvind Vilas Gadekar ${ }^{6}$, MBBS, Yong Mong ${\underline{B e e^{2}}}$, MBBS, MRCP; IO HAT Investigator group

INTRODUCTION Hypoglycaemia constitutes a significant barrier to achieving glycaemic control with insulin in both Type 1 (T1DM) and Type 2 diabetes mellitus (T2DM). The International Operations Hypoglycaemia Assessment Tool (IO HAT) study was designed to determine the incidence of hypoglycaemia in insulin-treated patients with T1DM and T2DM. METHODS The IO HAT study retrospectively and prospectively assessed the incidence of hypoglycaemia in patients with insulin-treated diabetes mellitus in nine countries. This sub-analysis included patients from Singapore with T1DM or T2DM who were aged $\geq 21$ years and had completed two self-assessment questionnaires (SAQ1 and SAQ2).

RESULTS Of the 50 T1DM and 320 T2DM patients who completed the SAQ1, 39 T1DM and 265 T2DM patients completed SAQ2; $100 \%$ and $90.9 \%$, respectively, experienced at least one hypoglycaemic event prospectively. The incidence rates of any hypoglycaemia were 49.5 events per patient-year (EPPY) and 16.1 EPPY for T1DM and T2DM patients, respectively, in the four-week prospective period. Hypoglycaemia rate did not differ in terms of glycated haemoglobin level. The vast majority of T1DM or T2DM patients ( $92.0 \%$ and $90.7 \%$, respectively) knew the overall definition of hypoglycaemia before study participation, although over half of the patients (T1DM 54.0\%, T2DM 51.9\%) defined hypoglycaemia based only on symptoms.

CONCLUSION High proportions of insulin-treated patients with diabetes mellitus in Singapore reported hypoglycaemic events prospectively, showing that they had underreported hypoglycaemic episodes retrospectively. Patient education can help in improving hypoglycaemia awareness and its management in the region.

Keywords: diabetes, hypoglycaemia, insulin, IO HAT, Singapore

\section{INTRODUCTION}

The prevalence of diabetes mellitus (DM) in Singapore is rapidly increasing. ${ }^{(1,2)}$ The International Diabetes Federation's 2015 report showed that there were 541,500 cases of DM in Singapore in 2015 alone. ${ }^{(3)}$ In a report published by the Ministry of Health $(\mathrm{MOH})$ Singapore in 2010, DM was found to be the second most important cause of disability-adjusted life years, a parameter to assess the burden of disease. ${ }^{(4)}$

One of the barriers to glycaemic control in both Type 1 DM (T1DM) and Type 2 DM (T2DM) is the potential hypoglycaemia that occurs with therapeutics. ${ }^{(5,6)}$ Understanding the risk factors of hypoglycaemia in the specified population is the key to safer diabetic treatment for patients. Studies have identified various predictive factors associated with the risk of hypoglycaemia, including age, duration of DM, duration of insulin use, intensive glycaemic therapy, previous history of severe hypoglycaemia, renal impairment and alcohol consumption. ${ }^{(7,8)}$

In Singapore, the $\mathrm{MOH}$ has issued clinical practice guidelines for clinicians for the management of DM. ${ }^{(9)}$ The guidelines recommend educating insulin-treated patients with DM regarding hypoglycaemia management and self-monitoring of blood glucose (SMBG). Clinicians also need to be vigilant with patients who have near-normal glycaemic levels, as they are at higher risk of hypoglycaemia. ${ }^{(9)}$ Fear of hypoglycaemia is quite high in insulin-treated patients with DM and has a negative effect on the emotional state and well-being of the patient. This may, in turn, hinder optimal glycaemic control by preventing intensification of insulin therapy by physicians. ${ }^{(10,11)} \mathrm{A}$ local study in 2008 assessing the cost of DM and its related complications over five years showed that severe hypoglycaemia significantly increased healthcare costs by SGD 757 within the first year. ${ }^{(12)}$ Additionally, the duration of hospitalisation is known to be higher in patients with DM who experience hypoglycaemia. ${ }^{(13)}$ Nevertheless, aside from limited reports from clinical trials, real-world data on the evaluation of health service utilisation or hospitalisation related to hypoglycaemic events is lacking. ${ }^{(14)}$ The seemingly high prevalence of hypoglycaemia and its associated economic burden necessitates a comprehensive reassessment of the real-world burden of hypoglycaemia in patients with DM in Singapore.

The non-interventional International Operations Hypoglycaemia Assessment Tool (IO HAT) study was designed

${ }^{1}$ Department of Endocrinology, SingHealth Polyclinics, ${ }^{2}$ Department of Endocrinology, Singapore General Hospital, ${ }^{3}$ Department of Endocrinology, National University Hospital, ${ }^{4}$ Department of Endocrinology, Tan Tock Seng Hospital, Singapore, ${ }^{5}$ Novo Nordisk Healthcare AG, Zurich, Switzerland, 6 Novo Nordisk Pharma Operations (BAOS) Sdn Bhd, Malaysia Correspondence: Dr Anand B Jain, Senior Medical Manager - Insulins, Novo Nordisk Health Care AG, Thurgauerstrasse 36, CH-8050 Zurich, Switzerland. ajai@novonordisk.com 
to assess the incidence of hypoglycaemia in patients with insulin-treated DM in Bangladesh, Colombia, Egypt, Indonesia, the Philippines, Singapore, South Africa, Turkey and the United Arab Emirates. ${ }^{(15)}$ The IO HAT study builds on findings from the global HAT (Hypoglycaemia Assessment Tool) study that was conducted in 24 countries. ${ }^{(16)}$ In this sub-analysis, we assessed the incidence of hypoglycaemia retrospectively and prospectively among insulin-treated patients in Singapore with T1DM or T2DM.

\section{METHODS}

The IO HAT study was a non-interventional, multicentre, six-month retrospective and four-week prospective international survey on hypoglycaemia among insulin-treated patients with DM. It was conducted using two-part self-assessment questionnaires (SAQ1 and SAQ2) and a patient diary (PD) (Box 1). In this sub-analysis, data on hypoglycaemia was collected from patients recruited between 13 January 2015 and 11 May 2015 across five sites in Singapore. The study was conducted in accordance with the 2007 Guidelines for Good Pharmacoepidemiology Practices and the 2013 Declaration of Helsinki. ${ }^{(17,18)}$ The protocol, informed consent form and other documents such as recruitment procedures were reviewed and approved by country-specific regulatory and ethics agencies. The trial was registered on ClinicalTrials. gov (NCT02306681). Study materials were translated into local languages, and the data obtained was translated back into English for analysis.

The survey population comprised ambulatory and literate Singaporean patients with T1DM or T2DM who: were $\geq 21$ years of age at baseline; were on insulin treatment (pre-mixed, shortacting and/or long-acting) for > 12 months; and had given informed consent before participating in the survey. Patients were enrolled randomly via consecutive sampling during a routinely scheduled clinical consultation with their healthcare provider at primary or secondary care centres in Singapore.

The primary endpoint of the survey was to evaluate the proportion of patients experiencing at least one hypoglycaemic event during the four-week prospective observational period. Secondary endpoints included determination of incidence rates (IRs) of all types of hypoglycaemia in the four weeks before (six months before baseline for severe hypoglycaemia) and four weeks after the baseline visit; the relationship between glycated haemoglobin ( $\mathrm{HbA} 1 \mathrm{c})$ at baseline ( $\mathrm{HbA} 1 \mathrm{c}$ level $<7.0 \%$, $7.0 \%-9.0 \%$ and $>9.0 \%$ ), insulin regimen (short-acting, longacting, pre-mix, and combination of short- and long-acting insulin) and hypoglycaemia, frequency of SMBG, patient knowledge and fear of hypoglycaemia; and the impact of hypoglycaemia on the healthcare system, work and/or studies.

The SAQ1 was used to record baseline demographics, DM-related complications and treatment information; evaluate knowledge of hypoglycaemia, hypoglycaemic unawareness and perceptions of hypoglycaemia; and record the history of severe hypoglycaemia over the previous six months and any (including nocturnal) hypoglycaemia over the previous four weeks. The SAQ2 was used to collect information on hypoglycaemia during the four weeks' prospective period, and the effect of
Box 1. International Operations Hypoglycaemia Assessment Tool study design:

\section{Part 1: Retrospective (before baseline visit)}

- Cross-sectional evaluation.

- History of severe hypoglycaemia over the previous six months and history of non-severe hypoglycaemia over the previous four weeks was collected using the self-assessment questionnaire 1 (SAQ1).

- SAQ1 was returned on Day 1 (baseline visit).

Part 2: Prospective (after baseline visit)

- Observational evaluation.

- Information on hypoglycaemia in the four weeks after baseline was collected using the SAQ2.

- The SAQ2 was returned on Day 28.

- Patients used patient diary to record hypoglycaemic events during the four weeks after baseline.

hypoglycaemia on productivity and healthcare utilisation during this time frame. Patients were also provided with PDs to record any hypoglycaemic events occurring in the four weeks after baseline and to assist recall.

Patients who returned any part of any SAQ or PD containing answers to any of the questions received were also included in the full analysis set. Patients' knowledge of hypoglycaemia was assessed by checking if their definition was consistent with American Diabetes Association's hypoglycaemia definition ${ }^{(19)}$ and if they were aware of hypoglycaemia before reading the introduction provided in the informed consent form. Hypoglycaemia unawareness was evaluated with an SAQ question, 'Do you have symptoms when you have a low sugar level?', where the response 'Usually' denoted impaired awareness, and 'Occasionally' or 'Never' denoted severely impaired awareness (unawareness). ${ }^{(20)}$ Fear of hypoglycaemia was rated by the patient on a scale of 0 (not afraid at all) to 10 (absolutely terrified).

Hypoglycaemia, as documented in the SAQ and PD, was classified as (a) non-severe hypoglycaemia: documented symptomatic (symptoms and blood glucose measurement $\leq 3.9 \mathrm{mmol} / \mathrm{L}$ [70 mg/dL]) and probable symptomatic (symptoms only) hypoglycaemia; (b) severe hypoglycaemia: an event requiring the assistance of another person to actively administer carbohydrate, glucagon or other resuscitative actions (based on the American Diabetes Association's hypoglycaemia definition); ${ }^{(19)}$ (c) nocturnal hypoglycaemia: an event occurring between midnight and $6.00 \mathrm{am}$; and (d) any hypoglycaemia: an aggregate index of all hypoglycaemic events from any of the categories.

The incidence of hypoglycaemia was compared in the retrospective and prospective periods using two-sided tests, in association with the DM type and insulin regimen used. Statistical significance was set at $p<0.05$. For the primary endpoint, the proportion of patients who experienced at least one hypoglycaemic episode during the four-week prospective observational period among T1DM or T2DM was calculated together with the $95 \%$ confidence interval $(\mathrm{Cl})$. Incidence rates of hypoglycaemia were reported as events per patient-year (EPPY), calculated as the total number of events divided by total follow-up time in patient-years along with $95 \% \mathrm{Cl}$. Relationship 
Table I. Patient baseline characteristics.

\begin{tabular}{|c|c|c|c|c|c|}
\hline \multirow[t]{2}{*}{ Characteristic } & \multicolumn{2}{|c|}{ Mean \pm SD/no. (\%) } & \multirow[t]{2}{*}{ Characteristic } & \multicolumn{2}{|c|}{ Mean \pm SD/no. (\%) } \\
\hline & $\begin{array}{l}\text { T1DM } \\
(n=50)\end{array}$ & $\begin{array}{l}\text { T2DM } \\
(n=320)\end{array}$ & & $\begin{array}{l}\text { T1DM } \\
(n=50)\end{array}$ & $\begin{array}{l}\text { T2DM } \\
(n=320)\end{array}$ \\
\hline Age (yr) & $41.2 \pm 13.8$ & $58.6 \pm 11.8$ & Glucagon-like peptide-1 analogues & $0(0)$ & $2(0.6)$ \\
\hline Gender* & & & Meglitinides/glinides & $0(0)$ & $1(0.3)$ \\
\hline Male & 54.0 & 51.3 & SGLT2 inhibitors & $0(0)$ & 35 (10.9) \\
\hline Duration of DM (yr) & $18.4 \pm 11.9$ & $18.0 \pm 9.2$ & Thiazolidinediones/glitazones & $0(0)$ & $0(0)$ \\
\hline Duration of insulin use (yr) & $16.8 \pm 11.4$ & $6.6 \pm 5.9$ & Other & $0(0)$ & $3(0.9)$ \\
\hline HbA1c (\%) & $8.2 \pm 1.6$ & $8.8 \pm 1.8$ & None & $40(80.0)$ & $51(15.9)$ \\
\hline Fasting blood glucose (mmol/L) & $9.3 \pm 4.0$ & $8.5 \pm 3.4$ & Missing & $0(0)$ & $1(0.3)$ \\
\hline Postprandial glucose (mmol/L) & $11.7 \pm 5.7$ & $11.0 \pm 3.8$ & \multicolumn{3}{|l|}{ Insulin treatment $^{\dagger}$} \\
\hline Previous medical illness* & & & Long-acting & $0(0)$ & $77(24.1)$ \\
\hline Neuropathy & 4.0 & 22.2 & Pre-mixed & $10(20.0)$ & $155(48.4)$ \\
\hline Retinopathy & 16.0 & 24.1 & Both short- and long-acting & $38(76.0)$ & $61(19.1)$ \\
\hline Nephropathy & 10.0 & 25.6 & Both short-acting and pre-mixed & $0(0)$ & $16(5.0)$ \\
\hline Peripheral vascular disease & 2.0 & 8.1 & Both long-acting and pre-mixed & $0(0)$ & $2(0.6)$ \\
\hline Angina & 4.0 & 8.4 & Missing & $0(0)$ & $2(0.6)$ \\
\hline Myocardial infarction & 0 & 9.7 & \multicolumn{3}{|c|}{ Check blood sugar with own glucose meter ${ }^{\dagger}$} \\
\hline Cerebrovascular accident & 2.0 & 2.8 & Yes & $37(74.0)$ & $254(79.4)$ \\
\hline Transient ischaemic attack & 2.0 & 3.4 & No & $11(22.0)$ & $60(18.8)$ \\
\hline Angioplasty & 2.0 & 8.8 & Not sure & $2(4.0)$ & $4(1.3)$ \\
\hline Coronary artery bypass graft & 0 & 6.6 & \multicolumn{3}{|c|}{ Symptom of DM-related complications ${ }^{t, \neq}$} \\
\hline None & 72.0 & 32.5 & Any & $50(100.0)$ & $271(84.7)$ \\
\hline Alpha-glucosidase inhibitors & $0(0)$ & $17(5.3)$ & Dizziness & $32(64.0)$ & $161(50.3)$ \\
\hline Metformin & $10(20.0)$ & $242(75.6)$ & Weakness & $34(68.0)$ & $156(48.8)$ \\
\hline Dipeptidyl peptidase-IV inhibitors & $0(0)$ & $57(17.8)$ & Tiredness & $33(66.0)$ & $154(48.1)$ \\
\hline
\end{tabular}

*Data presented as percentage. †Percentages were calculated based on number of patients with evaluable data. $\neq$ Only top 5 symptoms are presented. DM: diabetes mellitus; HbA1c: glycated haemoglobin; SD: standard deviation; SGLT2: sodium-glucose co-transporter-2; T1DM: Type 1 DM; T2DM: Type 2 DM

between $\mathrm{HbA1c}$ at baseline and the log-transformed number of events for patients experiencing hypoglycaemia were shown on a scatter plot (data not presented) with regression line and 95\% $\mathrm{Cl}$. Continuous and categorical data were summarised using descriptive statistics and frequency tables (number of patients and percentage), respectively. Baseline data refers to data collected using the SAQ1, while follow-up data refers to data collected using the SAQ2 and, where applicable, PD.

\section{RESULTS}

A total of 370 patients (50 T1DM, 320 T2DM) were enrolled from study sites in Singapore and completed the SAQ1. Of these, 304 patients (39 T1DM, 265 T2DM) completed the SAQ2 and 307 patients (39 T1DM, 268 T2DM) completed the PD.

The baseline characteristics of the survey population are presented in Table I. Patients with T1DM were younger than those with T2DM (mean age 41.2 years vs. 58.6 years, respectively). The duration of insulin usage for patients with T1DM was considerably longer than that of patients with T2DM (16.8 years vs. 6.6 years, respectively). Overall, in the T1DM and T2DM pooled population, $66.4 \%$ of patients used insulin analogues, while $33.6 \%$ used human insulins. The mean $\mathrm{HbA} 1 \mathrm{c}$ level was $8.2 \%(66 \mathrm{mmol} / \mathrm{mol})$ and $8.8 \%(73 \mathrm{mmol} / \mathrm{mol})$ in patients with T1DM and T2DM, respectively.

In the four-week prospective period, the proportion of patients reporting at least one hypoglycaemic event was $100.0 \%(95 \% \mathrm{Cl}$ 91.0\%-100.0\%) with T1DM and 90.9\% (95\% Cl 86.7\%-94.1\%) with T2DM, while in the four-week retrospective period, $53.1 \%$ (95\% Cl 38.3\%-67.5\%) of patients with T1DM and 20.4\% (95\% Cl 16.1\%-25.3\%) of patients with T2DM experienced at least one hypoglycaemic event. In patients with T1DM, the estimated IR of any hypoglycaemia was significantly higher in the prospective period than in the retrospective period (49.5 [95\% Cl 41.9-58.2] EPPY vs. 18.4 [95\% Cl 14.3-23.2] EPPY, p < 0.001) (Fig. 1a). Similarly, in patients with T2DM, the IR of any hypoglycaemia was significantly higher in the prospective than the retrospective period (16.1 [95\% Cl 14.4-18.0] EPPY vs. 5.4 [95\% Cl 4.6-6.5] EPPY, $\mathrm{p}<0.001$; Fig. 1b).

In nocturnal hypoglycaemia, the proportion of patients reporting a hypoglycaemic event in the retrospective period was 

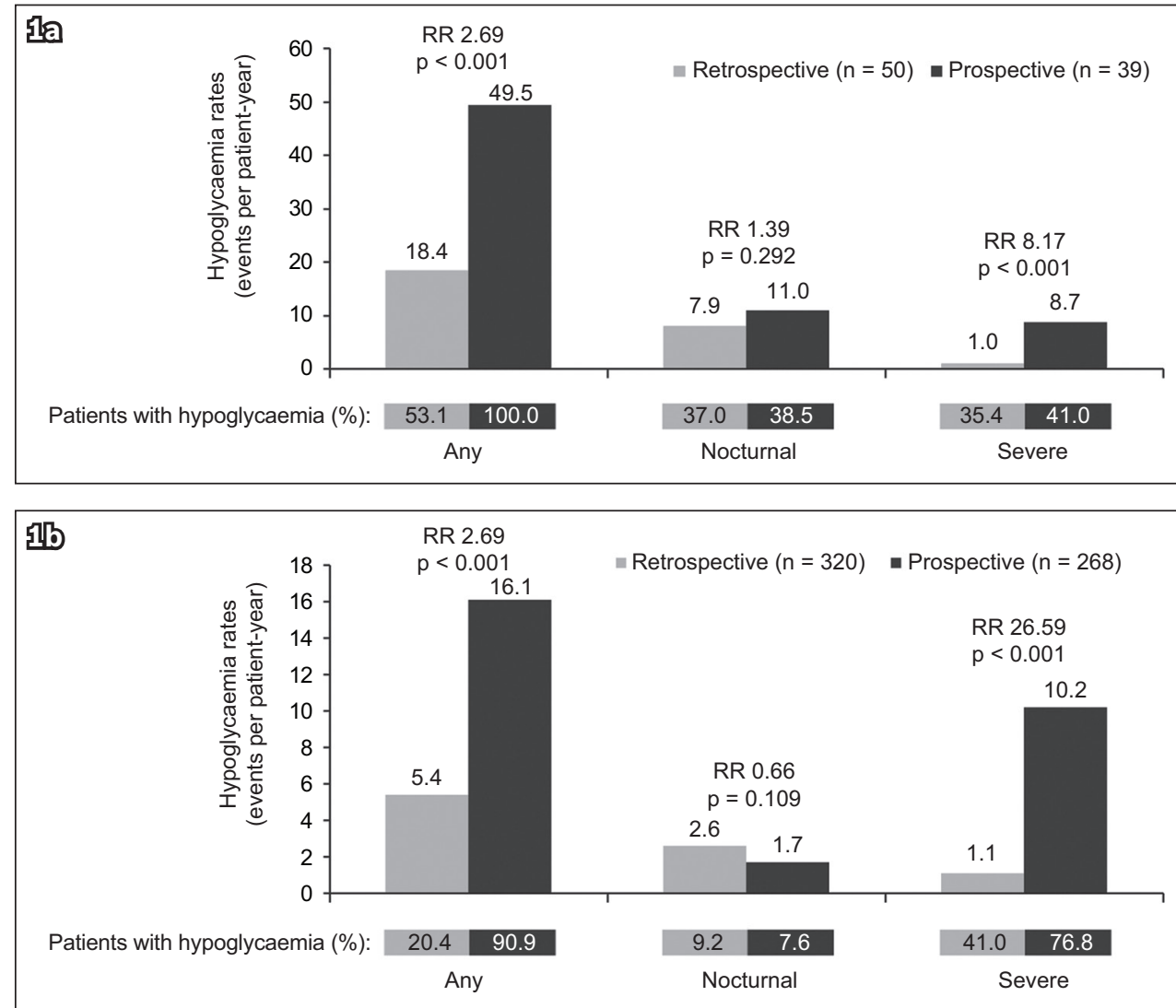

Fig. 1 Bar graphs show retrospective and prospective hypoglycaemic rates in patients with (a) Type 1 and (b) Type 2 diabetes mellitus. For any or nocturnal hypoglycaemia, data was based on a four-week period for both analyses. For severe hypoglycaemia, retrospective data was based on a six-month period and prospective data was based on a four-week period. RR: rate ratio

$37.0 \%(95 \% \mathrm{Cl} 23.2 \%-52.5 \%)$ and $9.2 \%(95 \% \mathrm{Cl} 6.3 \%-13.0 \%)$ in T1DM and T2DM, respectively, whereas $38.5 \%(95 \% \mathrm{Cl}$ $23.4 \%-55.4 \%)$ with T1DM and $7.6 \%(95 \% \mathrm{Cl} 4.7 \%-11.5 \%)$ with T2DM experienced at least one hypoglycaemic event in the prospective period. In the patients with T1DM, there was no significant difference in the estimated IR of nocturnal hypoglycaemia between the prospective period and the retrospective period (11.0 [95\% Cl 7.6-15.5] EPPY vs. 7.9 [95\% Cl 5.3-11.5] EPPY, $p=0.292$; Fig. 1a). Similarly, in patients with T2DM, there was no significant difference between the prospective period and the retrospective period (1.7 [95\% Cl 1.2-2.4] EPPY vs. 2.6 [95\% Cl 2.0-3.3] EPPY, $p=0.109$; Fig. 1b).

In T1DM, 35.4\% (95\% Cl 22.2\%-50.5\%) of patients in the sixmonth retrospective period and $41.0 \%(95 \% \mathrm{Cl} 25.6 \%-57.9 \%)$ of patients in the four-week prospective period experienced a severe hypoglycaemic event. The IR of severe hypoglycaemia was significantly higher in the prospective period compared to the retrospective period (8.7 [95\% Cl 5.7-12.7] EPPY vs. 1.0 [95\% Cl 0.7-1.6] EPPY, p < 0.001) (Fig. 1a). In T2DM, 41.0\% $(95 \% \mathrm{Cl} 35.4 \%-46.7 \%)$ of patients in the retrospective period and $76.8 \%(95 \% \mathrm{Cl} 71.1 \%-81.8 \%)$ of patients in the prospective period experienced a severe hypoglycaemic event, and the IR of severe hypoglycaemia was also significantly higher in the prospective period compared to the retrospective period (10.2 [95\% Cl 8.8-11.7] EPPY vs. 1.1 [95\% Cl 0.9-1.3] EPPY, $\mathrm{p}<0.001$; Fig. 1b).
The impact of hypoglycaemic events on the medical system during the retrospective and prospective periods is presented in Table II. None of the patients with T1DM needed an additional clinical appointment during the retrospective or prospective periods. However, $7.7 \%$ of T1DM patients made additional telephone contact as a result of hypoglycaemia in the retrospective period and $0.4 \%$ of T1DM patients experienced a hypoglycaemic event that resulted in hospital admission in the prospective period. Similarly, $7.0 \%$ of patients with T2DM made additional telephone contact during the retrospective period, compared to $1.1 \%$ in the prospective period (Table II).

A total of $82.0 \%$ patients with T1DM and $47.8 \%$ patients with T2DM were students or full- or part-time employees. In the retrospective period, more patients with T1DM than those with T2DM experienced hypoglycaemic events that resulted in absence from work or studies ( $7.3 \%$ vs. $2.6 \%$, respectively), late arrival to work or studies $(7.3 \%$ vs. $2.0 \%$, respectively), or early departure from work or studies ( $4.9 \%$ vs. $2.0 \%$, respectively; Table II).

Different actions were taken by patients in response to hypoglycaemic events (Fig. 2). In the retrospective period, the majority of patients either consulted their doctor/nurse or sought medical assistance after experiencing hypoglycaemia (T1DM: 38.0\% in both categories; T2DM: 20.3\% and 20.9\%, respectively). In the prospective period, the majority of T1DM patients either increased their calorie intake (23.1\%) or increased blood glucose monitoring (25.6\%). Very few T2DM patients took action in response to hypoglycaemia. 


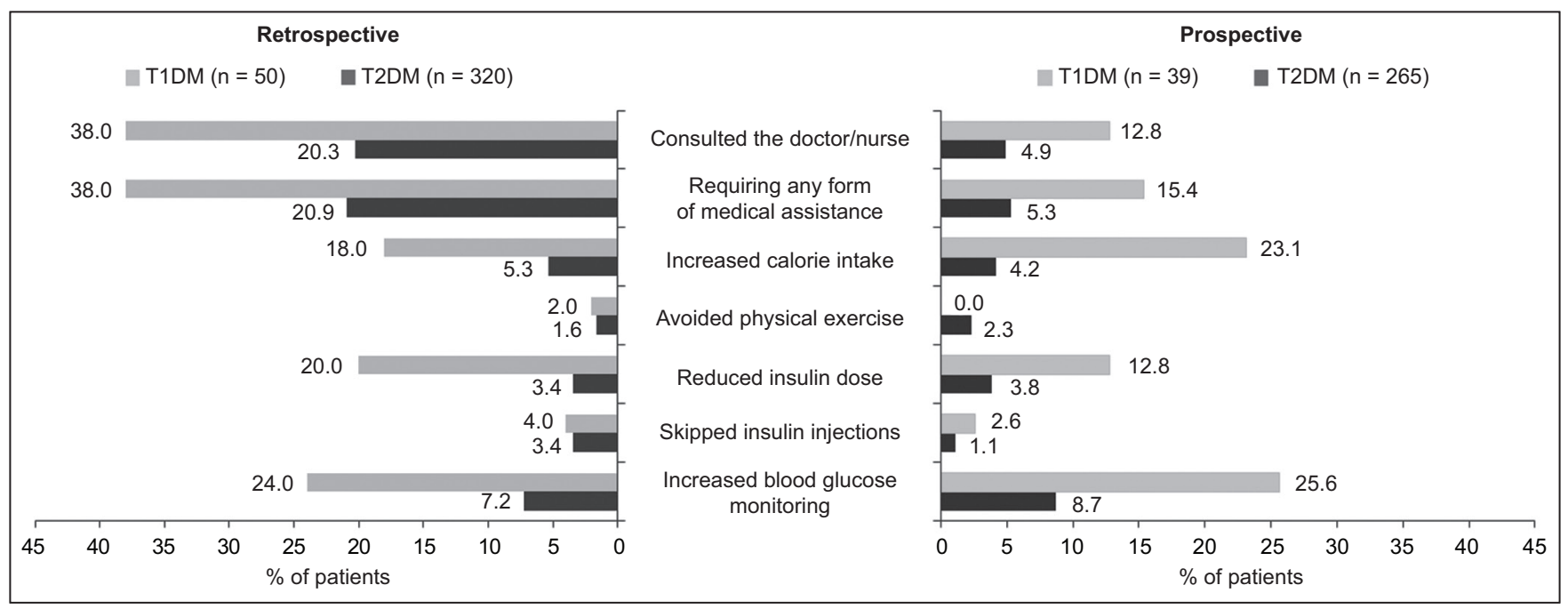

Fig. 2 Graph shows patient actions resulting from hypoglycaemia, based on the proportion of patients who responded 'Yes' to each question. T1DM: Type 1 diabetes mellitus; T2DM: Type 2 diabetes mellitus

Table II. Impact of hypoglycaemic events on medical system and work/studies.

\begin{tabular}{|llllll|}
\hline & \multicolumn{5}{c}{$\%$} \\
\cline { 2 - 3 } & \multicolumn{3}{c}{ Retrospective } & \multicolumn{2}{c|}{ Prospective } \\
\cline { 2 - 3 } & T1DM & T2DM & & T1DM & T2DM \\
\hline $\begin{array}{l}\text { Impact on medical } \\
\text { system }\end{array}$ & $(\mathrm{n}=39)$ & $(\mathrm{n}=186)$ & $(\mathrm{n}=39)$ & $(\mathrm{n}=264)$ \\
\hline $\begin{array}{l}\text { Events requiring } \\
\text { hospital admission }\end{array}$ & 0 & 0 & 2.7 & 0.4 \\
\hline $\begin{array}{l}\text { Attended additional } \\
\text { clinical appointments }\end{array}$ & 0 & 0 & 0 & 0 \\
\hline $\begin{array}{l}\text { Made additional } \\
\text { telephone contact }\end{array}$ & 7.7 & 7.0 & 0 & 1.1 \\
\hline $\begin{array}{l}\text { Impact on work and } \\
\text { studies }\end{array}$ & $(\mathrm{n}=41)$ & $(\mathrm{n}=153)$ & $(\mathrm{n}=30)$ & $(\mathrm{n}=117)$ \\
\hline $\begin{array}{l}\text { Took leave } \\
\text { Arrived late }\end{array}$ & 7.3 & 2.6 & 3.3 & 0 \\
\hline Left early & 4.9 & 2.0 & 0 & 0 \\
\hline
\end{tabular}

Values of $\mathrm{n}$ represent the no. of patients who experienced hyperglycaemic events during the respective time period, were studying or in full- or part-time employment, and completed Part 1 of the self-assessment questionnaire. T1DM: Type 1 diabetes mellitus; T2DM: Type 2 diabetes mellitus

Patients' perspectives on hypoglycaemia are provided in Table III. It was observed that knowledge of hypoglycaemia was high prior to the administration of SAQ1 (T1DM 92.0\%, T2DM 90.7\%). The majority of patients in both groups defined hypoglycaemia on the basis of symptoms alone (T1DM 54.0\%, T2DM 51.9\%) and had impaired hypoglycaemia awareness (T1DM 56.0\%, T2DM 66.6\%). Mean scores for self-reported fear of hypoglycaemia were comparable between the two groups (T1DM $4.7 \pm 2.8$ vs. T2DM $3.2 \pm 3.2$; Table III).

For patients with T1DM, the overall IRs of any, nocturnal and severe hypoglycaemia were higher in the prospective period. The highest IRs of any hypoglycaemia were observed with the use of a combination of short- and long-acting insulin regimens (57.4 EPPY) in the prospective period. The IRs of nocturnal hypoglycaemia were also highest for patients using a combination of short- and
Table III. Patient perspectives on hypoglycaemia.

\begin{tabular}{|lll|}
\hline \multirow{2}{*}{ Parameter } & \multicolumn{2}{c|}{$\% /$ mean \pm SD } \\
\cline { 2 - 3 } & $\begin{array}{l}\text { T1DM } \\
(\mathbf{n}=\mathbf{5 0})\end{array}$ & $\begin{array}{l}\text { T2DM } \\
(\mathbf{n}=\mathbf{3 2 0})\end{array}$ \\
\hline Knew what hypoglycaemia was* & 92.0 & 90.7 \\
\hline Basis for definition of hypoglycaemia & \\
\hline Symptoms only & 54.0 & 51.9 \\
\hline Blood glucose measurement only & 6.0 & 4.4 \\
\hline Either & 10.0 & 3.1 \\
\hline Both & 28.0 & 25.0 \\
\hline Hypoglycaemia awareness ${ }^{\dagger}$ & & \\
\hline Normal & 36.0 \\
\hline Impaired & 56.0 & 12.5 \\
\hline Severely impaired & 0 & 66.6 \\
\hline Fear of hypoglycaemia ${ }^{*}$ & $4.7 \pm 2.8$ \\
\hline
\end{tabular}

*At baseline before reading the definition in self-assessment questionnaire 1 . tHypoglycaemia unawareness was evaluated through the self-assessment question: 'Do you have symptoms when you have a low sugar level?', where the response 'Usually'denoted impaired awareness, and'Occasionally'or'Never'denoted severely impaired awareness (unawareness). $\neq$ On a scale of $0-10$. SD: standard deviation; T1DM: Type 1 diabetes mellitus; T2DM: Type 2 diabetes mellitus

long-acting insulin regimens (11.7 EPPY) in the prospective period. The IRs for severe hypoglycaemia were the highest for shortacting insulin regimens (13.0 EPPY) in the prospective period and lowest for the combined short- and long-acting insulin regimens (1.1 EPPY) in the retrospective period. For patients with T2DM, the proportion of participants and estimated IRs for all types of hypoglycaemia by insulin regimen are shown in Fig. 3 .

In this study, no correlation was seen between the proportion of patients with hypoglycaemia and baseline $\mathrm{HbA} 1 \mathrm{c}$ in the retrospective and prospective periods. For patients with T1DM, in the four-week retrospective period, the majority (88.9\%) of any hypoglycaemic events were experienced by patients in the good glycaemic control group ( $\mathrm{HbA} 1 \mathrm{c}<7.0 \%$ ), but the proportion of patients experiencing any hypoglycaemic events was higher in patients with poorly controlled glycaemia ( $\mathrm{HbA} 1 \mathrm{c}>9.0 \%)$ than in those with suboptimal glycaemic control (HbA1c 7.0\%-9.0\%) 

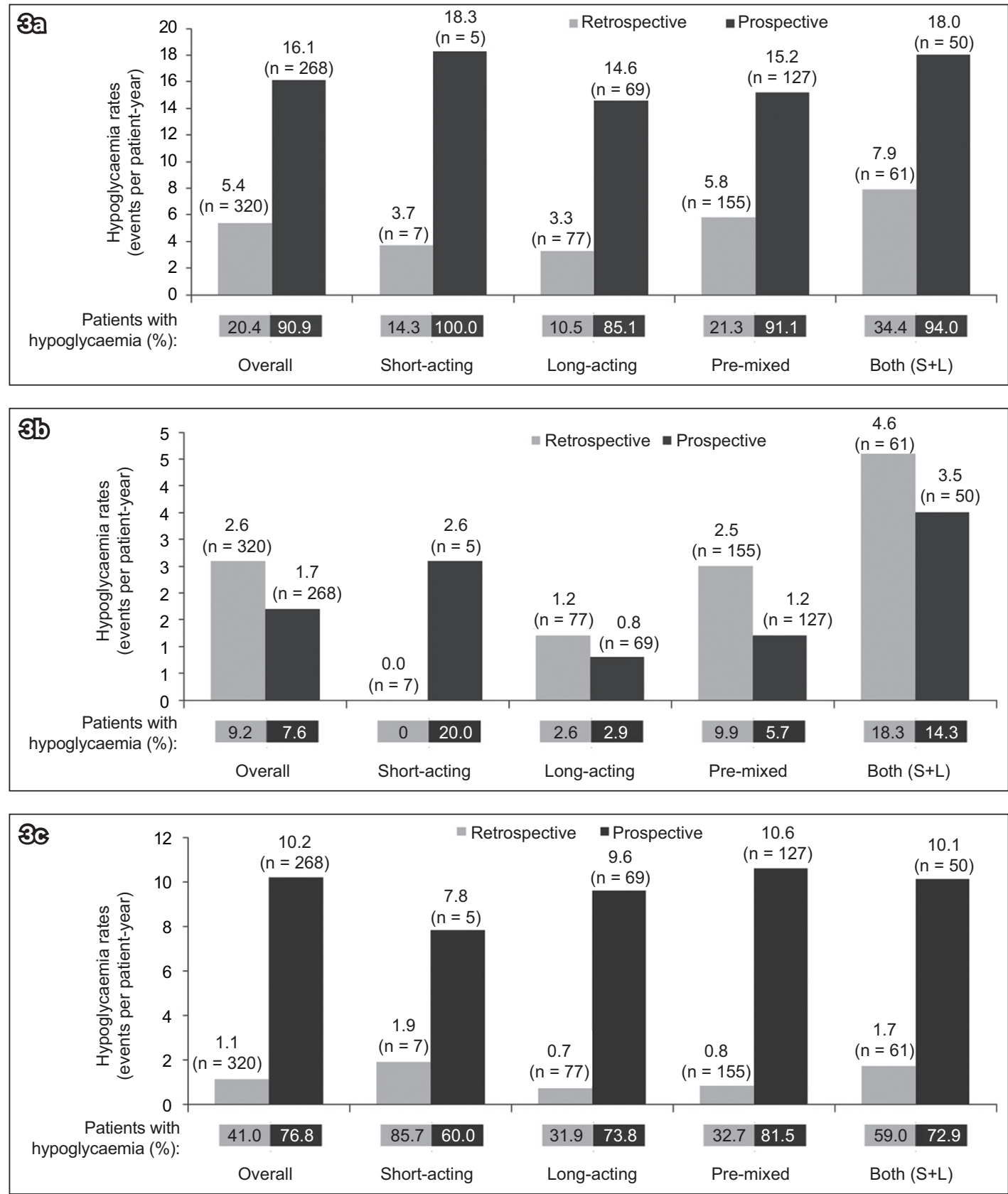

Fig. 3 Bar graphs show hypoglycaemia incidence and event rate in patients with Type 2 diabetes mellitus, by insulin regimen: (a) any hypoglycaemia, (b) nocturnal hypoglycaemia and (c) severe hypoglycaemia. For any or nocturnal hypoglycaemia, data was based on a four-week period for both analyses. For severe hypoglycaemia, retrospective data was based on a six-month period and prospective data was based on a four-week period. S+L: short-acting and long-acting insulin

(58.3\% vs. $39.3 \%$, respectively). For patients with T2DM, the proportions of patients in the three baseline $\mathrm{HbA1c}$ groups who had any hypoglycaemia were similar in the four-week retrospective period $(16.0 \%-27.3 \%)$.

\section{DISCUSSION}

This sub-analysis examined the prevalence of hypoglycaemic events in insulin-treated DM patients in the Singapore cohort of the IO HAT study. This dataset also provides information about knowledge and awareness of hypoglycaemia and its impact on the daily life of patients in Singapore.

The results showed that a higher percentage of patients reported hypoglycaemia in the prospective period compared to the retrospective period. This could be due to recall bias, since it is understandably more difficult to remember events retrospectively than prospectively, resulting in underreporting of events. In the prospective period, in addition to using the PD, patients are more likely to take note of possible hypoglycaemic symptoms. This recall bias was more pronounced for patients with severe hypoglycaemia, as they were required to retrospectively recall an event from the past six months; in contrast, the period of recall was just four weeks for any hypoglycaemia. The higher incidence of hypoglycaemia reporting in the prospective period could also be due to the SAQs and PDs, which served as tools of documentation and knowledge reinforcement. 
The IRs of any and severe hypoglycaemia were significantly lower in the retrospective period compared to the prospective period, showing that although patients had good knowledge of hypoglycaemia at baseline, this did not translate to accurate reporting of hypoglycaemic events. In practice, healthcare providers ought to be aware that their patients are likely to underreport hypoglycaemia based on recall alone. It would be a good practice to encourage patients to keep a diary of hypoglycaemic events for better documentation of events to facilitate further prevention and management.

Notably, nocturnal hypoglycaemia did not significantly differ between the assessment periods for patients with T1DM or T2DM. This might be because some of the patients had hypoglycaemia unawareness and thus reported less nocturnal hypoglycaemia than expected. Also, nocturnal hypoglycaemia (from midnight to $6.00 \mathrm{am}$ ) may go unnoticed and or unreported, as the events could occur during sleep hours, when the intensity and recognisability of counter regulatory responses tend to be diminished. (21) A slightly higher percentage of patients reported hypoglycaemia in the retrospective period than the prospective period ( $2.6 \%$ vs. $1.7 \%$ ) in the T2DM group. Patients may have reported higher incidences of nocturnal hypoglycaemia retrospectively when they were asked to recall, whereas having a clear definition of nocturnal hypoglycaemia in the prospective period allowed more accurate reporting during this period. Another perspective would be that nocturnal events may have impacted patients more, hence there was less recall bias.

Overall, for patients with T2DM, the rates of hypoglycaemia did not differ with the type of insulin regimen used. In T1DM, a high proportion of patients $(76 \%, \mathrm{n}=38)$ were on a combination of short- and long-acting insulins, followed by pre-mixed insulin $(20 \%, \mathrm{n}=10)$ and short-acting insulin $(4 \%, \mathrm{n}=2$; Table I). As expected, any hypoglycaemia was common with the most commonly used regimen (a combination of short- and long-acting insulin). However, the incidence of severe hypoglycaemia with short-acting insulin was skewed by data from one patient who had one episode of severe hypoglycaemia in the prospective period. The results of this study, especially from the T1DM group, can be used for physician education in the region. The focus should be on using insulin regimens with lower associated rates of hypoglycaemia, such as modern insulin analogues or the use of insulin pumps in conjunction with continuous glucose monitoring devices in patients with T1DM. ${ }^{(6,22,23)}$

Similar to findings from the primary IO HAT study, ${ }^{(15)}$ results from this cohort showed that the rate of any hypoglycaemic event was independent of $\mathrm{HbA} 1 \mathrm{c}$ levels at baseline. Therefore, hypoglycaemia can also occur in patients with higher $\mathrm{HbA} 1 \mathrm{c}$ levels. These results are in line with the findings from a recently published study, where HbA1c levels were not associated with risk of hypoglycaemia in an older population with T2DM who were on insulin therapy. ${ }^{(24)}$ Thus, it is important for healthcare providers to proactively screen patients for hypoglycaemia events regardless of their $\mathrm{HbA} 1 \mathrm{c}$ reading.

Patients in Singapore with T1DM or T2DM had good knowledge and were capable of identifying hypoglycaemia.
Despite this, the knowledge was not transferred to actual practice; $50 \%$ of the study population defined hypoglycaemia based on symptoms alone and only a quarter confirmed the symptoms with a blood glucose check. The majority of patients had impaired hypoglycaemia awareness, which indicates the importance of SMBG. SMBG can be a valuable focus as we enhance patient knowledge of the management of hypoglycaemia.

Goh et al, in their review of the use of insulin and risk of hypoglycaemia in the Southeast Asian population, reported that hypoglycaemic events result in increased medical complications, medical expenditure, loss of productivity and trauma in patients with DM. ${ }^{(25)}$ The fear of hypoglycaemia in such patients adversely affects glycaemic control by preventing intensification of insulin therapy or, in some cases, even resulting in discontinuation of insulin therapy. ${ }^{(25,26)}$ Results from the current study also demonstrate the substantial impact of hypoglycaemia on the healthcare system, work and productivity. Unfortunately, very few patients with T2DM took action in response to hypoglycaemic events, highlighting the need for patient counselling on recognition and management of hypoglycaemia.

Similar to the global HAT study, this sub-analysis was limited by its observational nature and short prospective duration. Recall bias associated with retrospective periods may be one of the limitations preventing direct comparison of prospective and retrospective periods. Potentially, the incidence of hypoglycaemia may be under- or overestimated due to self-reporting of data in the $\mathrm{PD}$ and SAQ2. Data obtained from the current study can pave the way for future research on managing hypoglycaemia in patients in Singapore, as it shows that patient education on hypoglycaemia and other factors such as regular SMBG and better tailoring of insulin treatment for patients with DM can help in minimising the risk of hypoglycaemia.

In conclusion, this study has shown the magnitude of hypoglycaemia among insulin-treated patients with T1DM and T2DM in Singapore. A high proportion of patients reported hypoglycaemic events with the use of questionnaires and PDs during the prospective period. However, healthcare providers ought to be aware that hypoglycaemic events can be underreported in insulin-treated patients locally. The majority of the patients are unlikely to confirm hypoglycaemic symptoms with blood glucose checks, and most T2DM patients would not take action. There is a need to focus on these areas when educating patients in managing hypoglycaemia. It is also important to explore ways to improve documentation of hypoglycaemia events as they occur. Accurate reporting and appropriate management of hypoglycaemia can improve the overall well-being of the patient.

\section{REFERENCES}

1. Phan TP, Alkema L, Tai ES, et al. Forecasting the burden of type 2 diabetes in Singapore using a demographic epidemiological model of Singapore. BMJ Open Diabetes Res Care 2014; 2:e00012.

2. Lee WR. The changing demography of diabetes mellitus in Singapore. Diabetes Res Clin Pract 2000; 50 Suppl 2:S35-9.

3. International Diabetes Federation. IDF Diabetes Federation Western Pacific. Singapore. Available at: http://www.idf.org/membership/wp/singapore. 
Accessed April 17, 2017.

4. Ministry of Health, Singapore. Singapore burden of disease study 2010 Available at: https://www.moh.gov.sg/content/dam/moh_web/Publications/ Reports/2014/Singapore\%20Burden\%20of\%20Disease\%20Study\%202010\%20 Report_v3.pdf. Accessed April 17, 2017.

5. Ahrén B. Avoiding hypoglycemia: a key to success for glucose-lowering therapy in type 2 diabetes. Vasc Health Risk Manag 2013; 9:155-63.

6. Kalra S, Mukherjee JJ, Venkataraman S, et al. Hypoglycemia: the neglected complication. Indian J Endocrinol Metab 2013; 17:819-34.

7. Childs BP, Grothe JM, Greenleaf PJ. Strategies to limit the effect of hypoglycemia on diabetes control: identifying and reducing the risks. Clin Diabetes 2012; 30:28-33.

8. Shafiee G, Mohajeri-Tehrani M, Pajouhi M, Larijani B. The importance of hypoglycemia in diabetic patients. J Diabetes Metab Disord 2012; 11:17.

9. Ministry of Health, Singapore. Diabetes mellitus. $\mathrm{MOH}$ clinical practice guidelines 1/2014. Available at:https://www.moh.gov.sg/content/dam/ moh_web/HPP/Doctors/cpg_medical/current/2014/diabetes_mellitus/cpg_ Diabetes\%20Mellitus\%20Booklet\%20-\%20Jul\%202014.pdf. Accessed May 2, 2017.

10. Sakane N, Kotani K, Tsuzaki K, et al. Fear of hypoglycemia and its determinants in insulin-treated patients with type 2 diabetes mellitus. J Diabetes Investig 2015 ; 6:567-70.

11. Gonder-Frederick. L. Fear of hypoglycemia: a review. Diabet Hypoglycemia 2013; 5:3-11.

12. Chow WL, Goh SY, Ying WS, Ng BKH, Lim JFY. Treatment of poorly controlled type 2 diabetes (T2DM) patients with insulin glargine--an economic analysis. Available at: http://www.davejunia.com/chsr/files/Conf_Treatment $\% 20$ of $\% 20$ poorly\%20controlled\%20type $\% 202 \% 20$ diabetes $\% 20($ T2DM) $\% 20$ patients $\% 20$ with $\% 20$ insulin $\% 20$ glargine $\% 20 \%$ E2\%80\%93\%20An $\% 20$ economic $\% 20$ analysis.pdf. Accessed May 2, 2017.

13. Ong KY, Kwan YH, Tay HC, Tan DS, Chang JY. Prevalence of dysglycaemic events among inpatients with diabetes mellitus: a Singaporean perspective. Singapore Med J 2015; 56:393-400.

14. Heng BH, Sun Y, Cheah JT, Jong M. The Singapore National Healthcare Group Diabetes Registry--descriptive epidemiology of type 2 diabetes mellitus. Ann Acad Med Singapore 2010; 39:348-52.

15. Emral R, Pathan F, Cortés CAY, et al; IO HAT Investigator group. Self-reported hypoglycemia in insulin-treated patients with diabetes: results from an international survey on 7289 patients from nine countries. Diabetes Res Clin Pract 2017; 134:17-28.

16. Khunti K, Alsifri S, Aronson R, et al. Rates and predictors of hypoglycaemia in 27585 people from 24 countries with insulin-treated type 1 and type 2 diabetes: the global HAT study. Diabetes Obes Metab 2016; 18:907-15.

17. International Society for Pharmacoepidemiology. Guidelines for Good Pharmacoepidemiology Practices (GPP). Initially issued: 1996. Revision 3 June, 2015. Available at: https://www.pharmacoepi.org/resources/policies/ guidelines-08027/. Accessed May 2, 2017.

18. World Medical Association. World Medical Association Declaration of Helsinki. Ethical Principles for Medical Research Involving Human Subjects. 64th WMA General Assembly, Brazil, October 2013. Available at: https://www.wma.net/ wp-content/uploads/2016/11/DoH-Oct2013-JAMA.pdf. Accessed May 2, 2017.

19. Workgroup on Hypoglycemia, American Diabetes Association. Defining and reporting hypoglycemia in diabetes: a report from the American Diabetes Association Workgroup on Hypoglycemia. Diabetes Care 2005; 28:1245-9.

20. Pedersen-Bjergaard U, Pramming S, Thorsteinsson B. Recall of severe hypoglycaemia and self-estimated state of awareness in type 1 diabetes. Diabetes Metab Res Rev 2003; 19:232-40.

21. Banarer S, Cryer PE. Sleep-related hypoglycemia-associated autonomic failure in type 1 diabetes: reduced awakening from sleep during hypoglycemia. Diabetes 2003; 52:1195-203.

22. Hartman I. Insulin analogs: impact on treatment success, satisfaction, quality of life, and adherence. Clin Med Res 2008; 6:54-67.

23. Diabetes management guidelines. American Diabetes Association (ADA) position statement: type 1 diabetes through the life span. Treatment recommendations. In: National Diabetes Education Initiative ${ }^{\mathrm{TM}}$ [online]. Available at: http://www. ndei.org/ADA-type-1-diabetes-guidelines.aspx.html\#Treatment. Accessed May 18, 2017.

24. Munshi MN, Slyne C, Segal AR, et al. Liberating A1C goals in older adults may not protect against the risk of hypoglycemia. J Diabetes Complications 2017; 31:1197-9.

25. Goh SY, Hussein Z, Rudijanto A. Review of insulin-associated hypoglycemia and its impact on the management of diabetes in Southeast Asian countries. J Diabetes Investig 2017; 8:635-45

26. Ross SA, Tildesley HD, Ashkenas J. Barriers to effective insulin treatment: the persistence of poor glycemic control in type 2 diabetes. Curr Med Res Opin 2011; 27 Suppl 3:13-20. 